\title{
Simultaneous broadband observations and high-resolution X-ray spectroscopy of the transitional millisecond pulsar PSR J1023+0038
}

\author{
F. Coti Zelati ${ }^{1,2,3}$, S. Campana ${ }^{3}$, V. Braito ${ }^{3}$, M. C. Baglio ${ }^{4,3}$, P. D’Avanzo ${ }^{3}$, N. Rea ${ }^{1,2,5}$, and D. F. Torres ${ }^{1,2,6}$ \\ ${ }^{1}$ Institute of Space Sciences (ICE, CSIC), Campus UAB, Carrer de Can Magrans s/n, 08193 Barcelona, Spain \\ 2 Institut d'Estudis Espacials de Catalunya (IEEC), 08034 Barcelona, Spain \\ ${ }^{3}$ INAF, Osservatorio Astronomico di Brera, Via E. Bianchi 46, 23807 Merate (LC), Italy \\ e-mail: cotizelati@ice.csic.es \\ ${ }^{4}$ New York University Abu Dhabi, PO Box 129188, Abu Dhabi, United Arab Emirates \\ 5 Anton Pannekoek Institute for Astronomy, University of Amsterdam, Postbus 94249, 1090-GE Amsterdam, The Netherlands \\ ${ }^{6}$ Institució Catalana de Recerca i Estudis Avançats (ICREA), 08010 Barcelona, Spain
}

Received 6 November 2017 / Accepted 30 December 2017

\begin{abstract}
We report on the first simultaneous XMM-Newton, NuSTAR, and Swift observations of the transitional millisecond pulsar PSR J1023+0038 in the X-ray active state. Our multi-wavelength campaign allowed us to investigate with unprecedented detail possible spectral variability over a broad energy range in the X-rays, as well as correlations and lags among emissions in different bands. The soft and hard X-ray emissions are significantly correlated, with no lags between the two bands. On the other hand, the X-ray emission does not correlate with the UV emission. We refine our model for the observed mode switching in terms of rapid transitions between a weak propeller regime and a rotation-powered radio pulsar state, and report on a detailed high-resolution X-ray spectroscopy using all XMM-Newton Reflection Grating Spectrometer data acquired since 2013. We discuss our results in the context of the recent discoveries on the system and of the state of the art simulations on transitional millisecond pulsars, and show how the properties of the narrow emission lines in the soft X-ray spectrum are consistent with an origin within the accretion disc.
\end{abstract}

Key words. methods: data analysis - methods: observational - stars: magnetic field - stars: neutron pulsars: individual: PSR J1023+0038 - X-rays: binaries

\section{Introduction}

According to the pulsar recycling scenario first proposed in the 1980s (Alpar et al. 1982; Radhakrishnan \& Srinivasan 1982; Taam \& van den Heuvel 1986; Bhattacharya \& van den Heuvel 1991; see also Tauris \& van den Heuvel 2006; Srinivasan 2010), millisecond radio pulsars (MSPs) are old pulsars that have been spun up to short spin periods after a Gyr-long phase of mass transfer in a low-mass X-ray binary system (LMXB). Repeated swings between an accretion-disk-dominated LMXB state and a rotation-powered MSP state were recently observed over a few weeks in the system IGR J18245-2452 (Papitto et al. 2013). This discovery demonstrated that these transitions are not an irreversible, long-lasting process, but can also occur on time scales compatible with those of the variations of the mass accretion rate onto the neutron star (NS). At the moment of writing, the class of these so-called transitional millisecond pulsars (TMPs) includes three confirmed members: IGR J18245-2452 (Papitto et al. 2013), PSR J1023+0038 (Archibald et al. 2009) and XSS J12270-4859 (Bassa et al. 2014).

Observed as an eclipsing 1.69-ms radio pulsar in a $4.75 \mathrm{~h}$ binary orbit between 2007 and 2013 (Archibald et al. 2013), PSR J1023+0038 (J1023) has been the subject of extensive multi-wavelength monitoring campaigns since 2013 June, when radio pulsations became undetectable at all orbital phases (Stappers et al. 2014), and double-peaked $\mathrm{H} \alpha$ emission lines - the signature of an accretion disc - were clearly observed in the optical spectrum (Halpern et al. 2013).

Radio-timing monitoring programs with different facilities failed to detect pulsations at the NS spin period following the transition to the accretion state (see Jaodand et al. 2016 and references therein). Imaging observations in the 1-18 GHz frequency range between 2013 November and 2014 April unveiled a highly variable emission on timescales as short as minutes, with a flat spectrum possibly related to synchrotron emission originating in material outflowing from the system (e.g. in the form of a weakly collimated partially self-absorbed jet; Deller et al. 2015). The gamma-ray luminosity of $\mathrm{J} 1023$ quintupled approximately at the same epoch at which the radio pulsations disappeared, and enhanced $0.1-300 \mathrm{GeV}$ emission has been recorded since then (Stappers et al. 2014; Deller et al. 2015; see also Torres et al. 2017 for a more detailed study). A search for steady emission above $300 \mathrm{GeV}$ in data acquired in 2013 December with the VERITAS gamma-ray observatory resulted instead in an upper limit for the luminosity $L_{>300 \mathrm{GeV}}<1.5 \times 10^{32} \mathrm{erg} \mathrm{s}^{-1}$ (adopting the parallax distance of $1.37 \mathrm{kpc}$ calculated by Deller et al. 2012) at the 95 percent confidence level (Aliu et al. 2016).

A NUSTAR observation in 2013 October led to an estimated luminosity of about $6 \times 10^{33} \mathrm{erg} \mathrm{s}^{-1}$ in the $3-79 \mathrm{keV}$ band, a factor of $\sim 8$ larger than that attained in the rotation-powered MSP state (Li et al. 2014; Tendulkar et al. 2014). Evidence for significant X-ray variability was detected in the Swift data sets on 
time scales of a few tens of seconds (Patruno et al. 2014), but also of days/weeks (Coti Zelati et al. 2014; Takata et al. 2014). However, a set of nine observations carried out with the larger collecting area of the X-ray instruments on board XMM-Newton between 2013 November and 2015 December have all revealed a puzzling trimodal behaviour of the system in the soft X-rays (0.3-10 keV): J1023 spends about 70-80 percent of the time in a stable "high" mode $\left(L_{X} \sim 3.4 \times 10^{33} \mathrm{erg} \mathrm{s}^{-1}\right)$, which unpredictably alternates to a "low" mode $\left(L_{X} \sim 5 \times 10^{32} \mathrm{erg} \mathrm{s}^{-1}\right)$. Sporadic flaring episodes are also observed - although not in all the observations - reaching luminosities $L_{X} \sim 10^{34} \mathrm{erg} \mathrm{s}^{-1}$ (e.g. Jaodand et al. 2016). The switches between these modes occur on a timescale of tens of seconds. Coherent X-ray pulsations at the NS spin period are detected only when the system is in the high mode (Archibald et al. 2015), and the phase-connected timing solution reported by Jaodand et al. (2016) shows that the average NS spin-down rate in the LMXB state is only about 27 percent larger than that observed during the rotation-powered MSP state. This strongly suggests not only that the pulsar spin-down mechanism has been operating since the formation of an accretion disc around the NS, but also that the outflow of material has increased the spin-down and largely prevails over the spin-up torque imparted by the accreting material.

Recent simultaneous Chandra and Karl G. Jansky Very Large Array observations showed an anti-correlated variability pattern between the X-ray and radio emissions: the X-ray low mode is accompanied by a temporally coincident radio brightening, while the X-ray high mode occurs in correspondence of steady low-level radio emission (Bogdanov et al. 2015).

The system became $\sim 3.5$ and $\sim 1$ mag brighter in the UV and optical bands, respectively, compared to the rotation-powered MSP state (Halpern et al. 2013; Patruno et al. 2014). Optical photometry revealed modulation of the emission at the $4.75 \mathrm{~h}$ orbital period (Coti Zelati et al. 2014; McConnell et al. 2015; Shahbaz et al. 2015), due to reprocessing of the X-ray emission by the heated face of the companion star (Papitto et al., in prep.). Flaring activity with rapid $(\$ 3 \mathrm{~s})$ transition timescales was observed both in the optical and near infrared bands (Hakala \& Kajava 2018). The first optical polarimetric study of J1023 was conducted by Baglio et al. (2016), who suggested that the measured linear polarization of $\sim 1$ percent in the system emission and its possible modulation at the orbital period could be due to Thomson scattering with electrons in the disc. This would exclude the presence of a well-structured jet at optical wavelengths. More recently, Hakala \& Kajava (2018) unveiled a significant anticorrelation between the optical flux and the degree of linear polarization. They also reported on the possible presence of an additional transient polarized emission component during the flares, and deduced changes in the disc emission during these episodes from $\mathrm{H} \alpha$ spectroscopy and Doppler tomography. These results led the authors to propose that Thomson scattering from matter ejected by the propelling magnetosphere might be the mechanism powering the optical flares. Optical pulsations at the NS spin period were detected in observations carried out in 2016 March, making J1023 the first optical MSP ever detected (Ambrosino et al. 2017). This discovery led the authors to favour a scenario in which the pulsed emission originates from synchrotron emission by relativistic electrons and positrons in the magnetosphere of a rotation-powered pulsar.

The wealth of data collected for J1023 has hence revealed unique properties of the system, and finding a unified scenario able of taking into account all of the above phenomenological characteristics of this system, as well as of the other transitional pulsars, has represented a tough challenge in the last years.
In a previous study we interpreted the repeated transitions between the high and low modes in terms of fast swings between the propeller regime and the rotation-powered MSP state (Campana et al. 2016; see also Linares 2014), and showed how this scenario could satisfactorily explain the multiwavelength properties of J1023 observed over the past 4 years. Here we present new simultaneous XMM-Newton, NuSTAR, and Swift observations which allowed us to search for correlations and lags between multiband emissions, as well as to improve our model for the observed mode switching of the system. We also present the results of our searches for narrow spectral features in archival Reflection Grating Spectrometer data with the aim of constraining the location of the emitting regions.

The study is structured as follows: we describe our multiwavelength observations and the data reduction in Sect. 2. We show the results of our searches for correlations and lags among the different energy bands in Sect. 3. We perform the first strictly simultaneous broadband characterisation of the different modes and refine our modelling for the mode switching in Sect. 4. We present a detailed characterisation of the high-resolution X-ray spectrum in Sect. 5. Discussion of the results and conclusions follow in Sect. 6.

\section{Observations and data extraction}

\subsection{XMM-Newton}

J1023 was observed with the European Photon Imaging Cameras (EPIC) on board XMM-Newton starting on 2016 May 8 at 03:29 UT and over one entire revolution (see Table 1 for details on the observation). The pn (Strüder et al. 2001) was set in fast timing (FT) mode, where data are read every $29.52 \mu$ s and only one-dimensional imaging is preserved. The two MOS cameras (Turner et al. 2001) were operated in small window mode (SW, $0.3 \mathrm{~s}$ time resolution) over the first $113 \mathrm{ks}$ of the observation. During the last $6.7 \mathrm{ks}$ segment of the observation, they both switched to a different observational setup and experienced serious telemetry issues, yielding a dramatic and unphysical drop of the source flux. In the following, we do not consider these data sets, and focus only on the longest uninterrupted exposures covering the first $113 \mathrm{ks}$ of the observation. The thin optical blocking filter was positioned in front of the three cameras. The Reflection Grating Spectrometer (RGS; den Herder et al. 2001) arrays were configured in standard spectroscopy mode ( $5.7 \mathrm{~s}$ time resolution) throughout the observation. The Optical/UV Monitor Telescope (OM; Mason et al. 2001) collected the data both in the "Image" and "Fast Window" modes, and used the $B$ filter, which is centred at $4392 \AA$ and covers the wavelength interval from 3800 to $5000 \AA$. Unfortunately, J1023 fell outside the small window of the instrument in the "Fast Window" mode data sets due to a small attitude problem (N. Schartel, priv. comm.). We thus focus on the image mode data alone. A total of 27 exposures were acquired, resulting in a total deadtime-corrected on source exposure time of $100 \mathrm{ks}$.

\subsubsection{Optical/UV Monitor Telescope data}

We reduced the data using the OMICHAIN processing pipeline with the default parameter settings, as recommended by the SAS threads. We determined the source magnitudes (reddened and in the Vega system) with OMSOURCE to extract the optical light curve, which is shown in Fig. 1. A fit with a sinusoidal function with period fixed to the orbital period of the binary system (17716 s; see Jaodand et al. 2016) yields $\chi_{v}^{2}=205.65$ 
Table 1. Log of the simultaneous observations of J1023 on 2016 May 8-9.

\begin{tabular}{|c|c|c|c|c|c|c|}
\hline Satellite & Obs. ID & Instrument & Mode & $\begin{array}{l}\text { Start - End time of exposure } \\
\text { (UTC) }\end{array}$ & $\begin{array}{l}\text { Exposure }^{a} \\
\quad(\mathrm{ks})\end{array}$ & $\begin{array}{l}\text { Source net count rate } \\
\quad\left(\text { counts s}^{-1}\right)\end{array}$ \\
\hline \multirow{5}{*}{ XMM-Newton } & \multirow{5}{*}{0784700201} & EPIC pn & FT & 2016/05/08 04:21:57 - 2016/05/09 14:46:27 & 121.7 & $3.641 \pm 0.008$ \\
\hline & & EPIC MOS 1 & SW & 2016/05/08 03:47:12 - 2016/05/09 12:08:48 & 112.9 & $1.001 \pm 0.003$ \\
\hline & & EPIC MOS 2 & SW & 2016/05/08 03:47:57 - 2016/05/09 12:09:02 & 113.0 & $1.004 \pm 0.004$ \\
\hline & & $\begin{array}{l}\text { RGS } 1 \\
\text { RGS } 2\end{array}$ & Spectroscopy & $\begin{array}{l}\text { 2016/05/08 03:47:02 - 2016/05/09 14:42:33 } \\
2016 / 05 / 08 \text { 03:47:10 - 2016/05/09 14:43:21 }\end{array}$ & $\begin{array}{l}125.4 \\
125.6\end{array}$ & $0.0893 \pm 0.0008$ \\
\hline & & OM & Image & 2016/05/08 07:49:52 - 2016/05/09 16:46:19 & 100.0 & - \\
\hline NUSTAR & 30201005002 & $\begin{array}{l}\text { FPMA } \\
\text { FPMB }\end{array}$ & Science & 2016-05-07 15:34:34 - 2016-05-09 15:13:26 & $\begin{array}{l}86.0 \\
85.7\end{array}$ & $\begin{array}{l}0.173 \pm 0.001(70 \%) \\
0.166 \pm 0.001(71 \%)\end{array}$ \\
\hline Swift & 0790180101 & UVOT & event & $2016 / 05 / 08$ 18:45:01 - 2016/05/09 12:21:57 & 9.5 & $0.90 \pm 0.01$ \\
\hline
\end{tabular}

Notes. ${ }^{(a)}$ Deadtime corrected on-source exposure. ${ }^{(b)}$ Background-subtracted count rate in the $0.3-10 \mathrm{keV}$ energy band for the EPIC instruments, in the $0.35-2.5 \mathrm{keV}$ energy band for the combined RGS first order data sets, in the 3-79 keV energy band for the NuSTAR data (the number in parentheses representing the fractional contribution from the 3-10 keV band), and in the UVM2 filter for the Swift UVOT data.

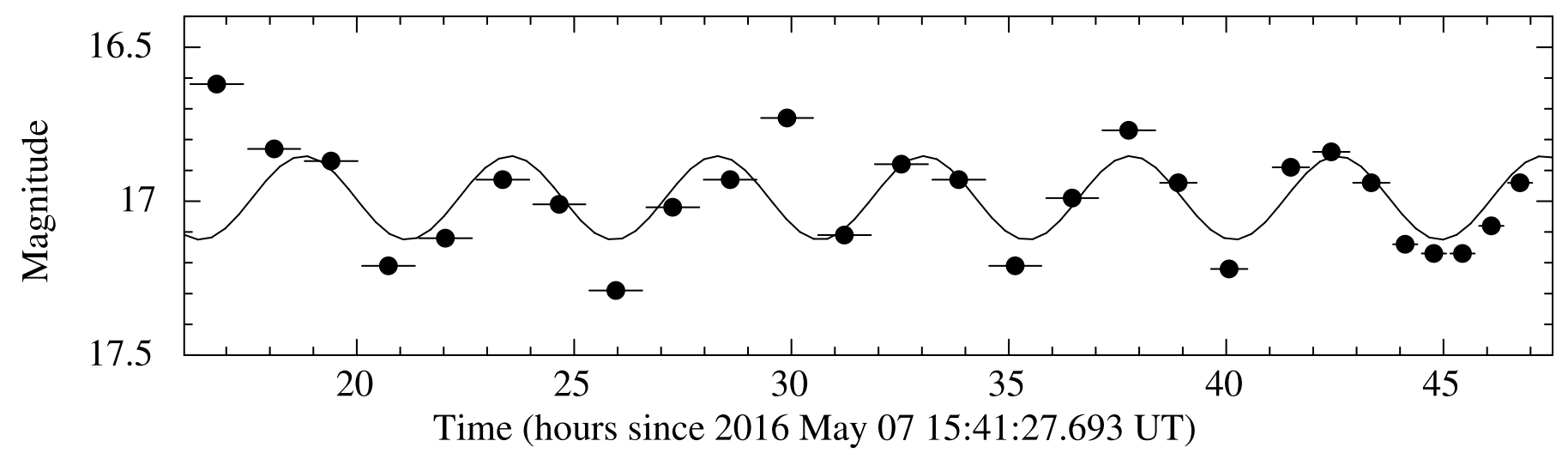

Fig. 1. Optical light curve of J1023 in the $B$ band. The solid line represents the best-fitting sinusoidal function (the period was fixed at the orbital period reported by Jaodand et al. 2016; see the text for details). Errors on the magnitudes are smaller than the size of the markers.

for 23 degrees of freedom (dof), a result that clearly hints at additional variability superimposed on any orbital modulation, possibly in the form of flaring episodes as in the X-rays. Unfortunately, a detailed study of this variability on shorter timescales (similarly to what is reported by Jaodand et al. 2016) is precluded since the fast-window mode data do not provide any useful information on the source because of the above mentioned loss of data.

\subsubsection{EPIC data}

We processed the raw observation data files using the $X M M-$ Newton Science Analysis System ( SAS $^{1}$ v. 15.0) and the most up to date calibration files available (XMM-CCF-REL-332).

We built a light curve of single-pixel events $($ PATTERN $=0)$ for the whole field of view and for each camera, to visually inspect for the presence of enhanced background flaring activity induced by soft-proton flares of solar origin. We revealed no significant contamination in all the three cameras throughout the observation, except for the last $10 \mathrm{ks}$ in the pn data sets, which were hence excluded from the following analysis.

For the pn camera we extracted the source counts within a 10-pixel-wide strip centred on the brightest column of CCD pixels $(\mathrm{RAW}-\mathrm{X}=37$ ), and oriented along the readout direction of the CCD. Background events were accumulated within a similar region sufficiently far from the position of the

1 See http://www. cosmos.esa.int/web/xmm-newton/sas source so as to minimize the contribution from the wings of the point spread function (PSF). For the two MOSs, source counts were collected within a circle centred on the position of the radio counterpart of the system $\left(\mathrm{RA}=10^{\mathrm{h}} 23^{\mathrm{m}} 47^{\mathrm{s}} .69\right.$, Dec $=00^{\circ} 38^{\prime} 40^{\prime \prime} .85$; Archibald et al. 2013) and with a radius of 36 arcsec. The background was extracted from a circle of the same size located on the same CCD and far from detector areas possibly contaminated by out-of-time events from the source or too near to the CCD edges. Photon arrival times of all event lists were referred to the solar system barycentre reference frame using BARYCEN and the DE-200 solar system ephemeris (which we adopt in the following for all data sets).

\subsubsection{RGS data}

We processed the data of each RGS instrument using the task RGSPROC, and produced calibrated and concatenated photon event lists, source and background spectra, and response matrices. We reduced the data also for all the other nine observations performed during the LMXB state (see Table 2 for a journal of these observations), and focused our analysis on the firstorder spectra alone, which have a higher number of counts. A total of about 134500 counts were collected by both RGSs in an exposure time of $\sim 580 \mathrm{ks}$.

The X-ray spectral properties of J1023 are remarkably similar among all XMM-Newton observations, both averaged (see e.g. Bogdanov et al. 2015) and in the different X-ray modes (Campana et al. 2016). We assumed that the line 
Table 2. Log of the XMM-Newton RGS observations of J1023 since the system transition to the X-ray active state.

\begin{tabular}{|c|c|c|c|c|}
\hline Obs. ID & Instrument & $\begin{array}{c}\text { Start - End time of exposure } \\
\text { (UTC) }\end{array}$ & $\begin{array}{l}\text { Exposure } \\
\quad(\mathrm{ks})\end{array}$ & $\begin{array}{l}\text { Source net count rate } \\
\left.\quad(\text { counts s })^{-1}\right)\end{array}$ \\
\hline \multirow{2}{*}{0720030101} & RGS 1 & 2013/11/10 17:21:55 - 2013/11/12 06:03:36 & 131.8 & $0.079 \pm 0.001$ \\
\hline & RGS 2 & 2013/11/10 17:29:01 - 2013/11/12 06:03:37 & 131.5 & $0.092 \pm 0.001$ \\
\hline \multirow{2}{*}{0742610101} & RGS 1 & 2014/06/10 03:44:53 - 2014/06/11 12:41:17 & 116.5 & $0.074 \pm 0.001$ \\
\hline & RGS 2 & 2014/06/10 03:45:01 - 2014/06/11 12:42:07 & 116.6 & $0.084 \pm 0.001$ \\
\hline \multirow{2}{*}{0748390101} & RGS 1 & 2014/11/21 18:32:36 - 2014/11/22 04:09:25 & 34.5 & $0.091 \pm 0.002$ \\
\hline & RGS 2 & 2014/11/21 18:32:44 - 2014/11/22 04:09:30 & 34.5 & $0.105 \pm 0.002$ \\
\hline \multirow{2}{*}{0748390501} & RGS 1 & 2014/11/23 18:24:42 - 2014/11/24 04:09:55 & 35.0 & $0.073 \pm 0.002$ \\
\hline & RGS 2 & 2014/11/23 18:24:50 - 2014/11/24 04:09:57 & 35.0 & $0.082 \pm 0.002$ \\
\hline \multirow{2}{*}{0748390601} & RGS 1 & 2014/11/28 21:31:11 - 2014/11/29 03:19:47 & 20.8 & $0.084 \pm 0.003$ \\
\hline & RGS 2 & 2014/11/28 21:31:18 - 2014/11/29 03:19:42 & 20.8 & $0.095 \pm 0.003$ \\
\hline \multirow{2}{*}{0748390701} & RGS 1 & 2014/12/17 15:45:29 - 2014/12/18 01:24:06 & 34.6 & $0.080 \pm 0.002$ \\
\hline & RGS 2 & 2014/12/17 15:45:37 - 2014/12/18 01:24:01 & 34.5 & $0.091 \pm 0.002$ \\
\hline \multirow{2}{*}{0770581001} & RGS 1 & 2015/11/11 19:36:35 - 2015/11/12 03:48:28 & 29.4 & $0.081 \pm 0.002$ \\
\hline & RGS 2 & 2015/11/11 19:36:43 - 2015/11/12 03:48:29 & 29.4 & $0.097 \pm 0.002$ \\
\hline \multirow{2}{*}{0770581101} & RGS 1 & 2015/11/13 03:30:32 - 2015/11/13 09:52:31 & 22.8 & $0.120 \pm 0.003$ \\
\hline & RGS 2 & 2015/11/13 03:30:40 - 2015/11/13 09:52:31 & 22.8 & $0.134 \pm 0.003$ \\
\hline \multirow{2}{*}{0783330301} & RGS 1 & 2015/12/09 01:03:46 - 2015/12/09 08:27:21 & 26.5 & $0.084 \pm 0.002$ \\
\hline & RGS 2 & 2015/12/09 01:03:53 - 2015/12/09 08:27:20 & 26.5 & $0.097 \pm 0.002$ \\
\hline \multirow{2}{*}{0784700201} & RGS 1 & 2016/05/08 03:47:02 - 2016/05/09 14:42:33 & 125.4 & $0.083 \pm 0.001$ \\
\hline & RGS 2 & 2016/05/08 03:47:10 - 2016/05/09 14:43:21 & 125.6 & $0.096 \pm 0.001$ \\
\hline
\end{tabular}

Notes. The RGS instruments were configured in the Spectroscopy mode in all observations. Count rates are quoted for the $0.35-2.5 \mathrm{keV}$ energy band.

properties do not vary among the observations, and combined all background-subtracted spectra of each RGS module using the RGSCOMBINE script. We then verified that the stacked spectra of each RGS module were in good agreement with each other, and combined them into a single spectrum (RGSCOMBINE appropriately accounts for the response matrices and backgrounds of the different spectrometers). We then rebinned the spectrum by a factor of 5 (corresponding to the full-width at half-maximum of the instrument), and limited the analysis to the 6.2-35.4 wavelength interval $(0.35-2 \mathrm{keV})$, where the first-order spectra are best calibrated. We verified that rebinning the spectrum by a factor of 3 did not yield different values for the line parameters.

\subsection{NUSTAR}

The Nuclear Spectroscopic Telescope Array mission (NuSTAR; Harrison et al. 2013) pointed its mirrors towards J1023 starting on 2016 May 7 at UT 15:34:34 and for a total net exposure time of about $86 \mathrm{ks}$ (see Table 1). We processed the event files using the script NUPIPELINE (v. 0.4.5) of the NuSTAR Data Analysis Software (NUSTARDAS, v.1.6.0, distributed along with HEASOFT v. 6.20), and the instrumental calibration files stored in CALDB v20160824. As recommended in the online NuSTAR Data Analysis Software Guide ${ }^{2}$, we cleaned the unfiltered event files with the standard depth correction, significantly reducing the internal high-energy background. We also removed passages through the South Atlantic Anomaly setting "SAAMODE=optimized". We adopted the latest version of the NUSTAR clock file available

\footnotetext{
2 Seehttps://heasarc.gsfc.nasa.gov/docs/nustar/ analysis/nustar_sw-guide.pdf
}

(v. 66) to correct for drifts of the spacecraft clock caused by changing thermal conditions within the spacecraft. We collected the source counts within a circular region of radius 50 arcsec, and background photons from a circle of radius 135 arcsec located as far away from the source as possible and on the same detector chip. This approach induces small systematic uncertainties in the background, which is known to change from chip to chip (e.g. Wik et al. 2014). Corrections to the photons' times of arrival were applied by means of the BARYCORR tool.

\subsection{Swift UVOT}

The Swift satellite observed J1023 for about $9.5 \mathrm{ks}$, starting on 2016 May 8 at 18:44:56 UT. In this study we focus on data acquired with the Ultraviolet and Optical Telescope (UVOT; Roming et al. 2005), since the energy band covered by the X-ray Telescope (XRT; Burrows et al. 2005) is fully covered by the EPIC cameras and the XRT provide a much poorer counting statistics, a coarser time resolution (2.5 s) and uneven sampling of the source variability with respect to the XMM-Newton data.

The UVOT was operated in event mode, yielding a minimum time resolution of about $11 \mathrm{~ms}$, and used the $U V M 2$ filter (central wavelength of $2246 \AA$; see Poole et al. 2008 and Breeveld et al. 2011 for more details on the filters). We converted the raw coordinate positions into detector and sky coordinates and removed hot pixels with the COORDINATOR and UVOTSCREEN scripts, respectively. We then extracted the source and background counts within XSELECT, using a circle of radius 5 arcsec to collect the source photons and a close-by circle of radius 20 arcsec for the background. Photon arrival times were referred to the solar system barycentre reference frame using the BARYCORR task and the satellite orbit ephemeris file. 
F. Coti Zelati et al. : Simultaneous multiband observations and high-resolution X-ray spectroscopy of J1023

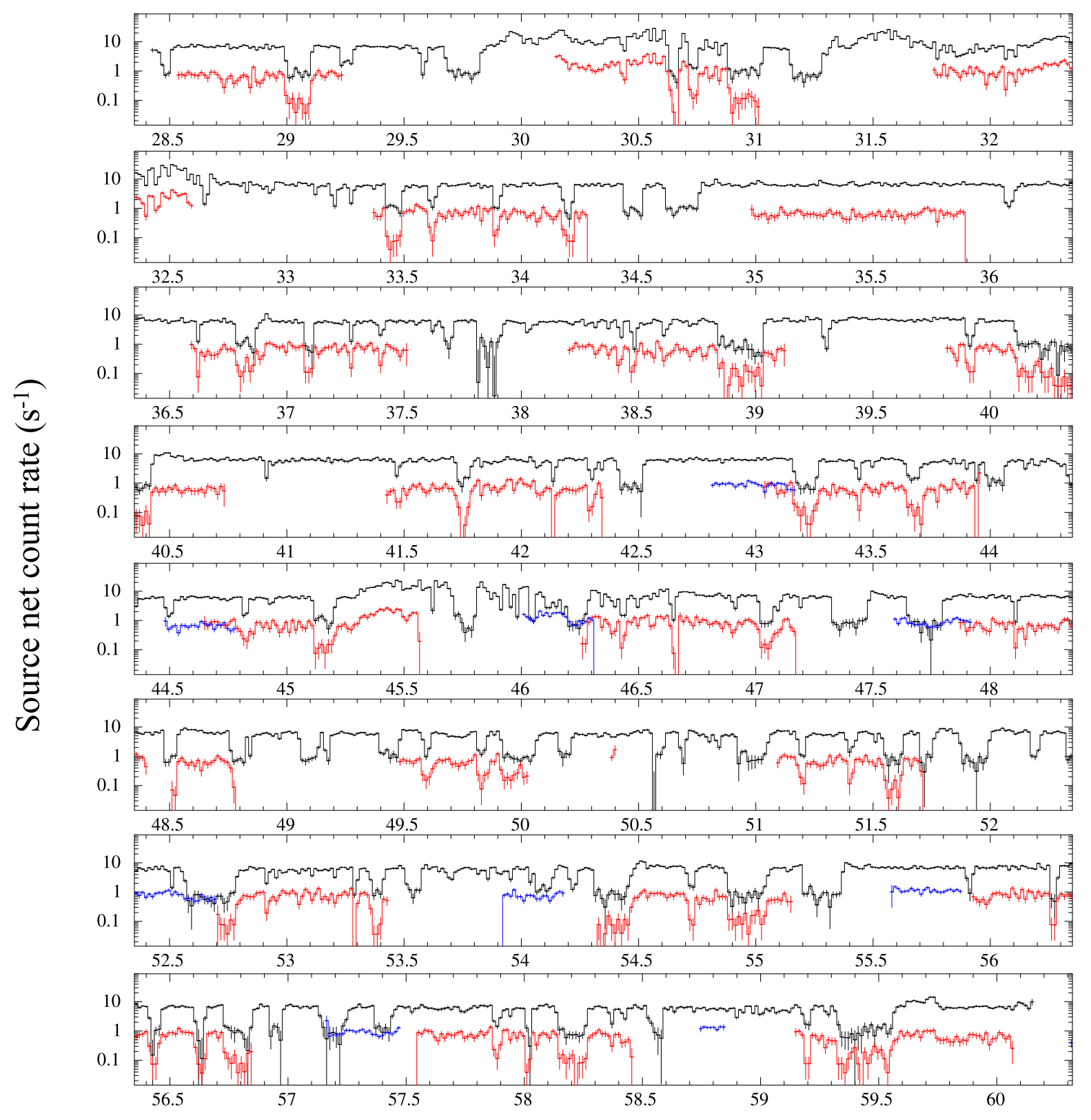

Time (hours since 2016 May 07 15:38:47.693 UT)

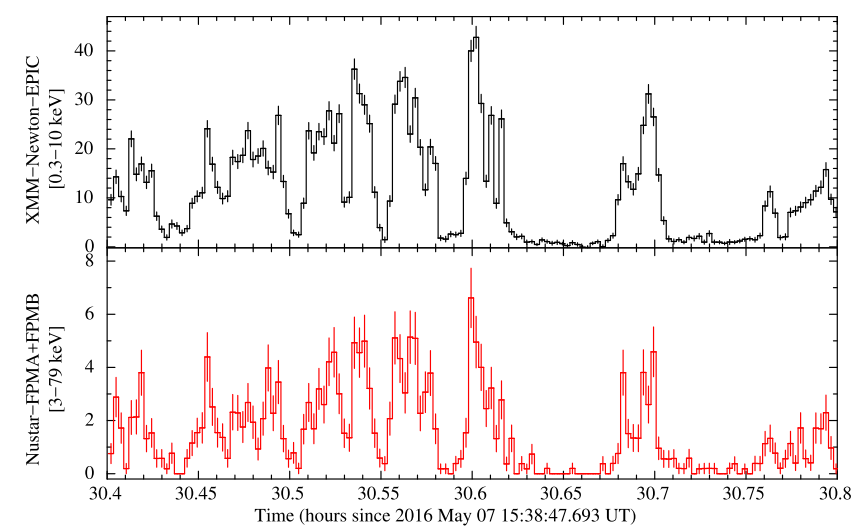

Fig. 2. Top: background-subtracted and exposure-corrected light curves of J1023 obtained with the XMM-Newton EPIC cameras (0.3-10 keV; black data), NuSTAR FPMA + FPMB (3-79 keV; red data) and Swift UVOT (UVM2 filter; blue data) during the time interval covered by XMM-Newton. For plotting purposes, light curves are shown with a binning time of $50 \mathrm{~s}$ and the vertical axis is plotted in logarithmic scale. Bottom: zoom of the $X M M-N e w t o n$ EPIC and NuSTAR FPMA + FPMB light curves around the longest and brightest flaring episode detected in the data. Light curves are shown with a binning time of $10 \mathrm{~s}$ and the vertical axis is plotted in linear scale. 


\section{Light curves, correlations, and lags}

The light curves of J1023 over the time interval covered by the XMM-Newton observation are reported in Fig. 2. Black, red, and blue data points refer to the XMM-Newton EPIC, NuSTAR FPMA+FPMB and Swift UVOT data sets, respectively. We first extracted the $0.3-10 \mathrm{keV}$ light curve from the XMM-Newton EPIC data sets by combining the $10 \mathrm{~s}$ binned backgroundsubtracted and exposure-corrected time series from each EPIC camera during the periods when all three telescopes acquired data simultaneously. Corrections for bad pixels, chip gaps, PSF variation, vignetting, quantum efficiency and dead time were also applied by means of the EPICLCCORR tool. We then built the 3-79 keV light curves from NUSTAR data separately for FPMA and FPMB using the NUPRODUCTS script, and combined them to increase the signal-to-noise ratio. Light curves were also extracted separately in the $3-10 \mathrm{keV}$ and $10-79 \mathrm{keV}$ energy bands. We then extracted the UV exposure-corrected and background-subtracted light curve for the Swift event lists by applying the LCMATH task, accounting for different areas of the source and background extraction regions.

The XMM-Newton and NuSTAR light curves display rapid and unpredictable variations in the count rate that are similar in all respects to those observed in all the previous XMM-Newton observations of the system while in its current state (Fig. 2; see also Bogdanov et al. 2015; Jaodand et al. 2016). For the $X M M-N e w t o n$ time series we found that, over the $32 \mathrm{~h}$ of the observation, the system spends about 3 percent of its time in a flaring mode (which we define for consistency with previous works as the mode where the source net count rate in the EPIC light curve binned at $10 \mathrm{~s}$ exceeds 15 counts $\left.\mathrm{s}^{-1}\right), \sim 66$ percent of the time in the "pure" high mode (count rate between 4 and 11 counts $\mathrm{s}^{-1}$ ), and $\sim 17$ percent of the time in the "pure" low mode (when the count rate drops below 2.1 counts $\mathrm{s}^{-1}$ ). In particular, we observe a sequence of three flaring events in the time series (between $\sim 29.8 \mathrm{~h}$ and $\sim 32 \mathrm{~h}$ in Fig. 2), followed by two additional episodes (between $\sim 45.3 \mathrm{~h}$ and $\sim 46.1 \mathrm{~h}$ in Fig. 2). During the brightest flares, the count rate in the EPIC light curves reaches values as high as $\sim 40$ counts $\mathrm{s}^{-1}$. The fractions of the time that the system spends in the different modes are remarkably similar to those observed in the other two deep XMM-Newton observations (Archibald et al. 2015; Bogdanov et al. 2015). We also note that J1023 spends a non-negligible ( $\sim 14$ percent) amount of time in an intermediate state between the "pure" high and low modes, related to the periods where the system switches between the two modes and during which the count rate attains a value outside the boundaries defined above. We computed the hardness ratio between soft $(0.3-10 \mathrm{keV})$ and hard (10-79 keV) X-ray bands, and found no substantial variation of the spectral shape across all modes.

We investigated possible time lags (on different timescales, from $0.1 \mathrm{~s}$ up to a few seconds) among the emission in the different energy bands using the task CROSSCOR of XRONOS. The shape of the cross-correlation function between the soft X-ray (0.3-10 keV, from XMM-Newton pn data) and hard X-ray (10-79 keV, from NuSTAR FPMA + FPMB data) is clearly symmetrical and shows no lags on the above-mentioned timescales (see the top panel of Fig. 3). We also verified that no lag is observed when considering the softest energy range in the pn data sets (i.e. below $3 \mathrm{keV}$ ), and also when analysing the emissions separately in the different modes. On the other hand, the X-ray and UV emissions are not correlated over the whole duration of the observations (see bottom panel of Fig. 3). Although a peak is visible at zero lag in the cross-correlation function,

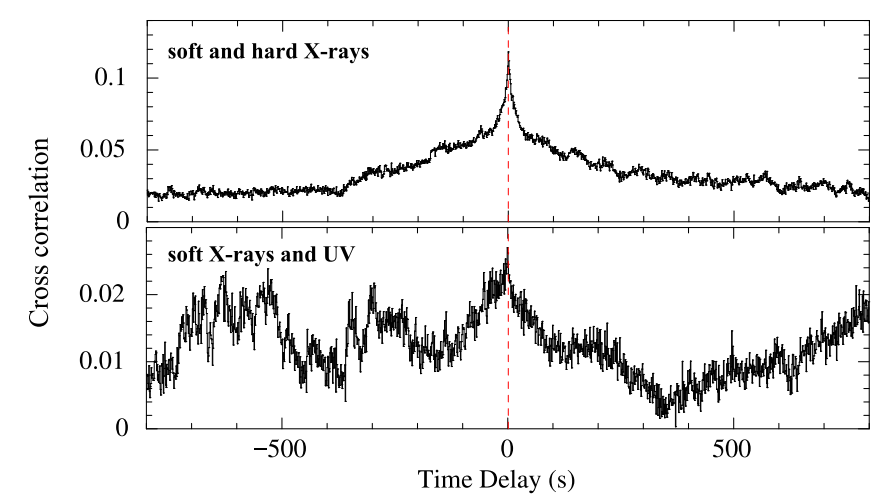

Fig. 3. Results of the cross-correlation between the time series in the 0.3-10 keV and 10-79 keV energy bands (top panel), and in the UV and the $0.3-10 \mathrm{keV}$ bands (bottom panel). In both panels lags are defined in such a way that positive time delays refer to the pn data with respect to FPM data (top), and to the pn data with respect to the UVOT data (bottom). In both panels the red dashed line marks the case of no time lag. We note the different interval on the vertical axis.

its significance is much lower than that of the peak measured between the soft and hard X-ray emissions, and comparable with that of other peaks (e.g. around $\sim-500 \mathrm{~s}$ ). These characteristics, as well as the overall asymmetric shape of the cross-correlation function, suggest that this peak is unlikely to reflect a true correlation. We note that some correlations and lags might be detected between X-rays and UV during the flares (as reported by Bogdanov et al. 2015 between the X-ray and optical bands). However, a multiband study of the system's flaring activity using our new data sets was precluded since Swift was not observing the source during the flaring episodes (see Fig. 2).

\section{Spectral analysis}

\subsection{Data extraction for the new observations}

Since we detected no lags between the XMM-Newton and the NUSTAR time series (see Sect. 3), we created the good time intervals associated to each of the three modes by applying the above-mentioned intensity thresholds to the EPIC light curve, that is, that characterised by the highest counting statistics (see also Bogdanov et al. 2015; Campana et al. 2016), and applied them to all our XMM-Newton and NUSTAR event files. The resulting exposure times and count rates for the $\mathrm{X}$-ray modes and the different X-ray instruments are listed in Table 3.

For the spectral analysis of EPIC data we retained only single- and double-pixel events (PATTERN $\leq 4)$ for the pn, and single to quadruple-pixel events for the MOSs (PATTERN $\leq 12$ ). Pixels and columns near the borders of the CCDs were excluded $($ FLAG $=0$ ). Redistribution matrices and ancillary files were built with RMFGEN and ARFGEN, respectively. For the NuSTAR data sets we extracted background-subtracted spectra and generated instrumental response files again separately for the two focal plane modules (FPMA and FPMB) with NUPRODUCTS.

For each instrument, events were retained within the energy interval whereby the calibration of the spectral responses is best known, that is, $0.3-10 \mathrm{keV}$ for the MOS, 0.7-10 keV for the pn (owing to known calibration uncertainties of this instrument at low energy in FT mode), and 3-79 keV for NuSTAR. Spectral channels were rebinned so as to contain a minimum of 200, 100, and 20 photons in each energy bin for the pn, MOS, and FPM data, respectively. 
Table 3. Log of the X-ray modes of J1023 from the simultaneous $X M M-N e w t o n$ EPIC and NuSTAR data sets.

\begin{tabular}{cccc}
\hline \hline Mode & Instrument & $\begin{array}{c}\text { Exposure } \\
(\mathrm{s})\end{array}$ & $\begin{array}{c}\text { Average count rate } \\
\left(\text { counts s }^{-1}\right)\end{array}$ \\
\hline \multirow{6}{*}{ Flare } & pn & 2613 & $13.94 \pm 0.07$ \\
& MOS 1 & 2533 & $3.92 \pm 0.04$ \\
& FPMA & 2400 & $3.83 \pm 0.04$ \\
& FPMB & 1676 & $0.85 \pm 0.02$ \\
High & pn & 52840 & $0.76 \pm 0.02$ \\
& MOS 1 & 55500 & $1.143 \pm 0.01$ \\
& MOS 2 & 51360 & $1.122 \pm 0.005$ \\
& FPMA & 37870 & $0.208 \pm 0.003$ \\
LPMB & 37660 & $0.200 \pm 0.002$ \\
\hline \multirow{4}{*}{ Fow } & pD & 16210 & $0.596 \pm 0.009$ \\
& MOS 1 & 17480 & $0.162 \pm 0.003$ \\
& FPMA & 15230 & $0.166 \pm 0.003$ \\
& FPMB & 11570 & $0.023 \pm 0.002$ \\
\end{tabular}

Notes. Count rates are in the same band as reported in Table 1.

\subsection{Spectral fits for all high- and low-mode spectra}

The spectral data sets of the new XMM-Newton and NuSTAR observations, together with those extracted consistently from all archival XMM-Newton and Chandra observations taken since 2008 (see Campana et al. 2016) allow us to refine our model for the high-low mode switching in terms of transitions between the weak propeller and radio pulsar regimes ${ }^{3}$. All spectra for the high and low modes were fitted together within XSPEC (Arnaud 1996) with the same model described by Campana et al. (2016): for the high mode we adopted a power law component associated with the propelling magnetosphere (see Papitto \& Torres 2015); a radiatively inefficient advection-dominated accretion disc (described with the DISKPBB model in XSPEC); a thermal component related to the X-ray pulsed emission arising from matter leaking through the propelling magnetosphere and accreting onto a restricted area of the NS surface (NSATMOS in XSPEC; see Heinke et al. 2006). For the low mode we included a power law with $\Gamma \sim 2$ to model the emission at the shock front between the relativistic pulsar particle wind and matter inflowing from the companion (see Tavani \& Arons 1997), again a radiatively inefficient accretion disc, and two components related to the radio pulsar activity: a thermal one originating from the NS polar caps (expected to be only slightly colder than during the high mode owing to the short timescale of the mode switching), and a nonthermal, power law-like one, related to magnetospheric mechanisms. The absorption by the interstellar medium along the line of sight was described via the TBABS model (Wilms et al. 2000).

In the spectral fits, we allowed the following parameters to vary: the disc temperature and inner radius, the polar cap temperature and radius, and the radio pulsar magnetospheric component. We required the disc temperature and inner radius to lie on the same $T(r) \propto r^{-p}$ curve across the two modes ( $p$ was always kept fixed to 0.5 ), and the polar cap radius to be the same across the two modes and smaller than the NS radius.

\footnotetext{
3 The bimodal shape of the X-ray flux distribution appears remarkably reproducible over a time span of years (2013-2017; see also Archibald et al. 2015; Bogdanov et al. 2015). Identical count rate thresholds were hence adopted to single out the high and low X-ray modes across all data sets.
}

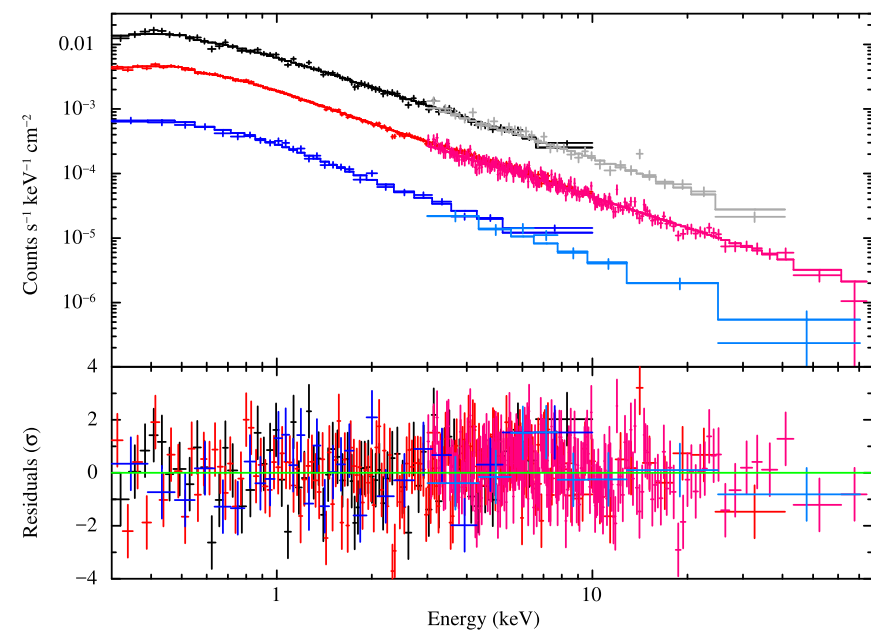

Fig. 4. Broadband X-ray spectra of J1023 relative to the three Xray modes from simultaneous XMM-Newton and NuSTAR data. For plotting purposes, only the XMM-Newton EPIC MOS1 (0.3-10 keV) and NuSTAR FPMA (3-79 keV) spectra are shown. Data have been re-binned to better visualise the trend in the spectral residuals. The bestfitting model is indicated by the solid lines. High- and low-mode spectra were fitted using the same model described by Campana et al. (2016; see Sect. 4.2), whereas flare spectra were fitted separately with an absorbed power law model (see Sect. 4.3). Black and grey refer to the flaring mode, red and magenta to the high mode, and blue and cyan to the low mode. Post-fit residuals in units of standard deviations are also shown in the bottom panel.

We tied up all parameters of the spectral components for the rotation-powered MSP state to those of the low mode. Finally, we tied up the hydrogen column density across all data sets.

We obtained a statistically acceptable result $\left(\chi_{v}^{2}=1.05\right.$ for 5246 d.o.f., after the addition of a systematic error of 2 percent; Smith 2015). We list the updated values for the parameters in Table 4, and for plotting purpose we show only the simultaneous XMM-Newton and NUSTAR spectra fitted with this model in Fig. 4, together with post-fit residuals.

\subsection{Spectral fits for the flaring mode spectra for the most recent observations}

We have additionally fitted an absorbed power law model to the XMM-Newton + NuSTAR spectra from the flaring mode, in order to detect possible variations in the spectral shape of this mode compared to that observed in previous observations. We fixed the column density to the value derived from the abovementioned model, that is, $N_{\mathrm{H}}=5.5 \times 10^{20} \mathrm{~cm}^{-2}$ (see Table 4) and obtained a photon index $\Gamma=1.67 \pm 0.01\left(\chi_{v}^{2}=1.07\right.$ for 255 d.o.f.). This is consistent with that observed up to $10 \mathrm{keV}$ in the long XMM-Newton observation in 2013 November (Bogdanov et al. 2015), implying no substantial variations in the spectral shape of the flaring mode on a timescale of $\sim 2.5 \mathrm{yr}$. We show the XMM-Newton and NuSTAR spectra with the best-fitting absorbed power law model in Fig. 4, together with post-fit residuals.

\section{High-resolution X-ray spectroscopy}

\subsection{Model for the continuum}

Before searching for narrow features in the RGS first-order spectrum, we extracted the stacked background-subtracted spectra from the MOS1 and MOS2 instruments from all observations 
Table 4. Results of the spectral modelling for the X-ray high and low modes and the rotation-powered MSP state of J1023 (see the text for details).

\begin{tabular}{cccc}
\hline \hline Spectral parameter & $\begin{array}{c}\text { High mode } \\
(319.2 \mathrm{ks})\end{array}$ & $\begin{array}{c}\text { Low mode } \\
(104.6 \mathrm{ks})\end{array}$ & $\begin{array}{c}\text { Rotation-powered MSP state } \\
(116.8 \mathrm{ks})\end{array}$ \\
\hline Power law $\Gamma_{D}$ & $1.58 \pm 0.01$ & $2.02 \pm 0.03$ & - \\
Power law $N_{D}\left(10^{-4}\right)$ & $17.6 \pm 0.4$ & $2.87 \pm 0.06$ & - \\
Disc $k T(\mathrm{eV})$ & $123_{-0.01}^{+0.02}$ & $<46$ & - \\
Disc norm. $N_{d}$ & $161_{-92}^{+155}$ & $>8 \times 10^{3}$ & tied to Low mode \\
NS atmos. $k T_{\infty}(\mathrm{eV})$ & $99_{-7}^{+9}$ & $50_{-4}^{+5}$ & tied to High mode \\
NS atmos. $R_{e m}(\mathrm{~km})$ & $3.5_{-0.6}^{+0.7}$ & tied to High mode & tied to Low mode \\
NS power law $\Gamma_{P}$ & - & $1.02 \pm 0.05$ & tied to Low mode \\
NS power law $N_{P}\left(10^{-5}\right)$ & - & $3.5 \pm 0.3$ & \\
\hline
\end{tabular}

Notes. The column density was tied up across all data sets, yielding $N_{\mathrm{H}}=5.5_{-0.6}^{+0.5} \times 10^{20} \mathrm{~cm}^{-2}$. The fit provides $\chi_{v}^{2}=1.05$ for 5246 d.o.f., after the addition of a systematic error of 2 percent. Errors are quoted at the $90 \%$ confidence level for one parameter of interest. The effective NS temperatures were corrected for the gravitational redshift: $k T_{\infty}=k T(1+z)^{-1}$ where $1+z=\left(1-2 G M_{N S} / R_{N S} c^{2}\right)^{-1 / 2}$ is the gravitational redshift factor, and $M_{N S}$ and $R_{N S}$ are the NS mass and radius. Radii were computed assuming a source distance of $1.37 \mathrm{kpc}$ (Deller et al. 2012).

carried out since the system transition to the LMXB state (see Table 1 by Jaodand et al. 2016 for a complete journal of these observations), to increase the counting statistics and better characterise the model for the continuum emission. The pn spectra were not considered for this purpose, owing to the known uncertainties in the spectral calibration of the data sets acquired in the timing mode at low energy (typically below $\sim 0.7 \mathrm{keV}$ ). The MOS spectra were grouped to have at least 100 counts in each spectral bin.

We started by fitting the stacked MOS1, MOS2, and RGS spectra simultaneously with a power law model corrected for the absorption by the Galactic interstellar medium (TBABS*POWERLAW), to adequately constrain the parameters for the continuum. The parameters were tied up across the three data sets, and a renormalization constant was included to account for intercalibration uncertainties. The choice of this simple phenomenological model to describe the continuum (compared to the more sophisticated one adopted for the different X-ray modes) was motivated by the fact that the power law component provides the largest contribution to the system's soft X-ray emission (see e.g. Fig. 3 by Campana et al. 2016). The counting statistics in the RGS spectrum is large enough to enable the use of the $\chi^{2}$ statistics to evaluate the goodness of the spectral fits (i.e. we verified that each spectral channel contained at least 20 photon counts). We obtained a photon index $\Gamma=1.82 \pm 0.02$ and absorption column density $N_{\mathrm{H}}=5.6_{-0.2}^{+0.3} \times 10^{20} \mathrm{~cm}^{-2}$, which is fully compatible within the uncertainties with the value reported in Sect. 4.2.

\subsection{Characterisation of the narrow features}

The poorer spectral resolution of the MOS instruments compared to the RGS precludes a detailed characterisation of narrow features in the EPIC data sets in the softest X-ray band. We hence focused on the RGS data, and fixed $N_{\mathrm{H}}$ and $\Gamma$ to the values derived from the simultaneous fit of the RGS and MOS data, that is, $N_{\mathrm{H}}=5.6 \times 10^{20} \mathrm{~cm}^{-2}, \Gamma=1.82$. We visually inspected structured residuals with respect to the model for the continuum in the RGS spectrum, and fitted the most significant residuals by including Gaussian components to the model, either in emission or absorption (progressively and in order of decreasing wavelength). Several complex structures were observed in the wavelength range encompassing the oxygen transitions (in particular in the 21-24 $\AA$ interval). We thus decided to substitute the TBABS model with the more physically motivated TBNEW_FEO model, which represents an improved high-resolution version of the Tuebingen-Boulder model (Wilms et al. 2000) where the absorption column for the oxygen and the iron are allowed to vary in the fit. This choice allowed us to obtain a sound modelling of the oxygen $\mathrm{K}$ edge and other features related to oxygen transitions. In fact, this component self consistently accounts for the strong $\mathrm{O}$ I $\mathrm{K} \alpha$ absorption line observed at $527.2 \mathrm{eV}$ (see Fig. 5). The abundances of the oxygen and iron were compatible with the solar values, and were hence fixed to those values. The widths of the Gaussian components were fixed to values smaller than the instrument energy resolution, that is, $\sigma=0$ or $1 \mathrm{eV}$.

The overall RGS spectrum can be satisfactorily described by including 16 Gaussian features (11 in emission and 5 in absorption). We obtained $\chi_{v}^{2}=0.92$ for 538 d.o.f. Table 5 reports the properties of the detected features as well as their identification with the transitions of the different elements. The expected energy of the transitions is also reported for comparison (see e.g. Engstrom \& Litzen 1995; Pradhan et al. 2003; Gu et al. 2005; Smith \& Brickhouse 2014). The significance for each feature (either in emission or absorption) is reported in terms of the improvement in the value of the $\chi^{2}$ when including the corresponding Gaussian component to the model, and as the ratio between the line normalization and the associated uncertainty at the $1 \sigma$ confidence level. The uncertainty on the line normalization was evaluated by allowing both the line centroid and normalization to vary in the fit. We also verified that the inclusion of additional spectral components to describe the continuum (NSATMOS and DISKPBB) did not significantly affect the properties of the narrow features (in particular their centroids and equivalent widths) and the significance of detection.

The spectrum with the best-fitting model superimposed is shown in Fig. 5 (both in the overall RGS wavelength coverage, as well as in selected wavelength ranges where the most prominent lines are detected). The H-like lines from $\mathrm{C} \mathrm{VI,} \mathrm{N} \mathrm{VII,} \mathrm{and}$ O VIII, as well as the C V, O II, O IV, O V, Fe XX, Fe XXIV, and $\mathrm{Mg}$ VII transitions are all detected close to, or compatible with, their expected rest frame energies or wavelengths. The line centroid energies measured for the N VI triplet of $424.7_{-0.7}^{+1.0} \mathrm{eV}$ and $429.4_{-1.7}^{+1.5}$ are consistent with the majority of the emission 
F. Coti Zelati et al. : Simultaneous multiband observations and high-resolution X-ray spectroscopy of J1023
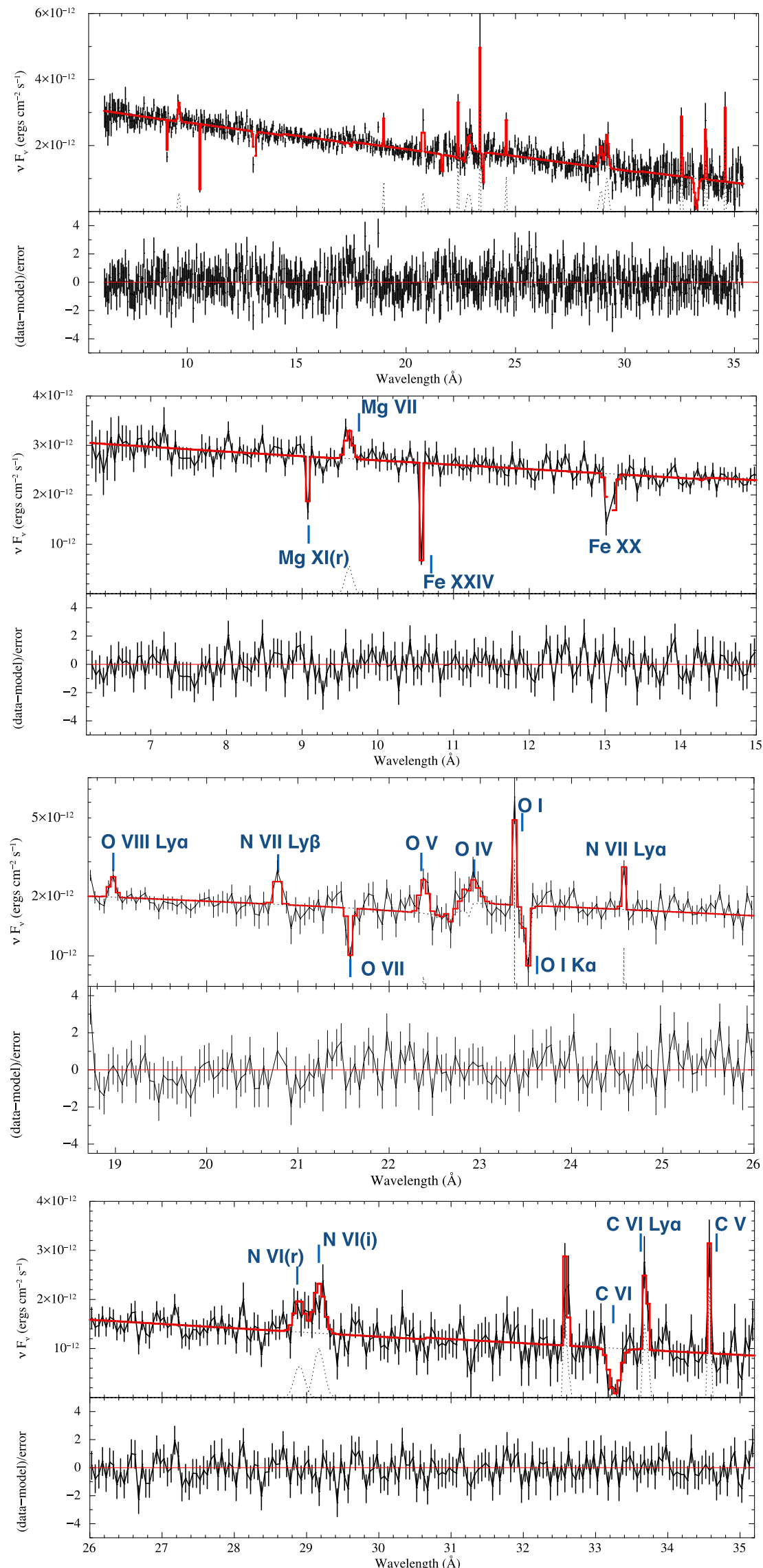

Fig. 5. Unfolded RGS first-order spectrum of J1023 in the 6.2-35.4 $\AA$ wavelength range (top panel) and in selected wavelength ranges (in order of increasing wavelength from top to bottom). The red solid line represents the best-fitting model (see the text for details). The wavelengths of the most relevant transitions are labelled in blue. Post-fit residuals are also shown. 
Table 5. Results of the spectral fits of the stacked RGS first-order spectrum of J1023.

\begin{tabular}{lccccccc}
\hline \hline Element transition & $\begin{array}{c}\text { Wavelength } \\
(\AA)\end{array}$ & $\begin{array}{c}\text { Energy } \\
(\mathrm{eV})\end{array}$ & $\begin{array}{c}\mathrm{E}_{l a b} \\
(\mathrm{eV})\end{array}$ & $|\Delta \lambda| / \lambda$ & $\begin{array}{c}\mathrm{EQW} \\
(\mathrm{eV})\end{array}$ & $\Delta \chi^{2}$ & $\begin{array}{c}\text { Significance } \\
(\sigma)\end{array}$ \\
\hline C V 1s-3p & 34.56 & $358.7 \pm 0.5(\mathrm{e})$ & 354.5 & 0.01 & $1.3_{-0.9}^{+1.1}$ & 4.62 & 2.0 \\
C VI Ly $\alpha$ & 33.69 & $368.0_{-1.6}^{+0.4}(\mathrm{e})$ & 367.5 & 0.001 & $1.4_{-1.4}^{+0.8}$ & 9.84 & 3.3 \\
C VI Ly $\alpha$ & 33.25 & $372.9 \pm 0.7(\mathrm{a})$ & 367.5 & 0.01 & $1.7_{-1.2}^{+1.2}$ & 7.53 & 3.1 \\
N VI (i) & 29.19 & $424.7_{-0.7}^{+1.0}(\mathrm{e})$ & 426.3 & 0.004 & $1.6_{-1.2}^{+0.8}$ & 11.24 & 3.4 \\
N VI (r) & 28.87 & $429.4_{-1.7}^{+1.5}(\mathrm{e})$ & 430.7 & 0.003 & $1.2_{-0.8}^{+1.1}$ & 6.01 & 2.7 \\
N VII Ly $\alpha$ & 24.56 & $504.8_{-2.0}^{+0.6}(\mathrm{e})$ & 500.3 & 0.009 & $0.7_{-0.4}^{+0.9}$ & 5.28 & 2.9 \\
O II 1s-2p & 23.38 & $530.4_{-0.3}^{+0.6}(\mathrm{e})$ & 532.9 & 0.005 & $1.9_{-1.2}^{+0.8}$ & 2.69 & 2.1 \\
O IV 1s-2p & 22.89 & $541.7_{-2.1}^{+2.3}(\mathrm{e})$ & 543.06 & 0.003 & $1.6_{-0.8}^{+1.1}$ & 4.84 & 2.3 \\
O V 1s-2p & 22.38 & $554.0_{-1.3}^{+1.7}(\mathrm{e})$ & 552.29 & 0.003 & $1.2_{-0.7}^{+0.7}$ & 7.60 & 2.7 \\
O VII 1s-2p & 21.57 & $574.7_{-0.5}^{+0.9}(\mathrm{a})$ & 573.95 & 0.001 & $0.8_{-0.4}^{+0.3}$ & 1.9 & 1.9 \\
Fe XX & 13.04 & $950.5_{-1.8}^{+1.2}(\mathrm{a})$ & 949.3 & 0.001 & $4.4_{-2.2}^{+2.0}$ & 16.95 & 3.9 \\
Fe XXIV & 10.56 & $1173.8_{-2.0}^{+2.1}(\mathrm{a})$ & 1167.6 & 0.005 & $4.1_{-1.7}^{+1.2}$ & 21.52 & 4.6 \\
Mg VII 1s-2p & 9.60 & $1291.0 \pm 4.1(\mathrm{e})$ & 1291.6 & 0.0005 & $3.2_{-1.7}^{+1.2}$ & 10.44 & 2.8 \\
Mg XI (r) & 9.07 & $1366.7_{-3.4}^{+1.7}(\mathrm{a})$ & 1357 & 0.007 & $2.9_{-1.9}^{+1.8}$ & 5.05 & 2.3 \\
\hline
\end{tabular}

Notes. Letters in parentheses indicate whether the narrow features are detected in emission (e) or absorption (a). EQW denotes the equivalent width of the Gaussian feature. Errors are reported at the 90 percent confidence level for a single parameter of interest. $\Delta \chi^{2}$ indicates the improvement in the overall fit when adding the Gaussian component to the model.

originating from the intercombination (near $426.3 \mathrm{eV}$ ) and resonance $(430.7 \mathrm{eV})$ transitions, respectively, but are inconsistent with the expected energy of the forbidden (at $419.8 \mathrm{eV}$ ) emission line. On the other hand, the line centroid energy measured at $1366.7_{-3.4}^{+1.7} \mathrm{eV}$ is close to the energy of the $\mathrm{Mg}$ XI resonance transition at an expected energy of $1357 \mathrm{eV}$.

We then tested whether it was possible to account for most of the emission lines using a more physically motivated collisionally ionized plasma model. We replaced the Gaussian lines with either a MEKAL or APEC component, as well as with the absorption grids 18 and 21 from the XSTAR library (Kallman et al. 1996) ${ }^{4}$. These cover similar ranges in both the ionization parameter $\left(\log \epsilon\right.$ from -4 to $\left.+4 \mathrm{ergs} \mathrm{cm} \mathrm{s}^{-1}\right)$ and column densities $\left(N_{\mathrm{H}}\right.$ from $10^{19}$ to $\left.10^{23} \mathrm{~cm}^{-2}\right)$, but assume significantly different turbulent velocities, 100 and $1000 \mathrm{~km} \mathrm{~s}^{-1}$ for the grid 18 and 21, respectively. Statistically acceptable fits were obtained in both cases by allowing the abundances of the different elements to vary $\left(\chi_{v}^{2}=1.05-1.10\right.$ for 577 d.o.f.). However, none of these models were able to reproduce properly the narrow features: visual inspection of the spectrum reveals structured residuals around most of the features. More complicated models are beyond the scope of this work.

We also performed an orbital phase-resolved spectral analysis of the system. We used the ephemerides calculated by Jaodand et al. (2016) to extract for each dataset the event files and spectra in three orbital phase bins of equal width $(0-0.33$, $0.33-0.66$ and $0.66-1)$. We then merged all data sets corresponding to the same phase bins. In the spectral analysis, all line parameters, the column density, and the power law photon index were kept fixed to their phase-averaged values, whereas the power law normalization was left free to vary to account for possible variations of the X-ray flux as a function of the orbital phase. We obtained acceptable fits in all cases, with $\chi_{v}^{2}$ ranging

\footnotetext{
4 See https://heasarc.gsfc.nasa.gov/lheasoft/xstar/ xstar.html
}

from 0.9 to 1.0. Visual inspection of the spectrum in restricted wavelength ranges revealed that the renormalized model provided an accurate modelling of the line shapes. We conclude that there are no statistically significant variations of the line properties along the orbital phase.

\section{Discussion and conclusions}

We report on the results of a simultaneous multi-wavelength observational campaign of the transitional millisecond pulsar J1023, encompassing the X-ray (XMM-Newton EPIC and NuSTAR), UV (Swift), and optical (XMM-Newton OM) bands. The deep $(\sim 120 \mathrm{ks})$ XMM-Newton observation revealed the expected trimodal behaviour of J1023 (i.e. high, low, and flaring mode) in its current state, showing intensity levels and timescales of switching remarkably similar to those observed in all the XMM-Newton data sets of the system acquired in the past 4 yrs. The NuSTAR data show a completely consistent variability at higher energy and on the same timescales (see Fig. 2).

The simultaneous XMM-Newton and NuSTAR observations of $\mathrm{J} 1023$ presented in this work allowed us to probe possible spectral hardening of the X-ray emission on an extended energy range. We do not observe significant spectral changes with energy in the different modes. J1023 shows some similarities with the other well-known transitional millisecond pulsar XSS J12270-4859 in the LMXB state. The detailed broad-band studies conducted by de Martino et al. (2010, 2013) indeed revealed that XSS J12270-4859 also does not show evidence for conspicuous spectral variability among the three modes, except for some hardening especially during the low-mode episodes following the flaring events.

Our multi-wavelength campaign allowed us to search for correlated variability and lags of the system emission at different wavelengths, including for the first time, the hard X-ray energy 
range. The cross-correlation function between the soft X-ray and hard X-ray emissions is characterized by a remarkably symmetric shape, and we do not observe any lag on the sampled timescales. Although the uneven sampling of NuSTAR and the lower counting statistics available especially at high energy precluded detailed characterisation of the shape of the crosscorrelation function during the different modes, inspection of the light curves binned at $10 \mathrm{~s}$ revealed a completely consistent behaviour in the soft and hard X-ray bands at the different luminosity levels. These results suggest that the bulk of the X-ray emission arises from the same physical mechanism. According to our model (see Campana et al. 2016, this should be associated to synchrotron radiation at the boundary between the propelling magnetosphere and the disk-mass in-flow in the high mode (see Papitto \& Torres 2015); synchrotron radiation in the local magnetic field at the shock front between the pulsar particle wind and the intra-binary material in the low mode (with negligible thermal contributions from the accretion disc and the pulsed emission from the NS surface during both modes; see Fig. 3 of Campana et al. 2016). Interestingly, de Martino et al. (2010, 2013) computed the cross-correlation function for the TMP XSS J12270-4859 in different soft X-ray $(<10 \mathrm{keV})$ bands during the LMXB state, and found a symmetric shape of the correlation function with no detectable time lag during both the flaring and low modes. These results are similar to what we report here for J1023 over a broader energy range (up to $79 \mathrm{keV}$ ) and also for the case of the high mode.

On the other hand, we do not observe any significant correlation between the X-ray and UV emissions during the high and low modes. This is consistent with the results reported by (Bogdanov et al. 2015), who performed cross-correlations between the X-ray and optical bands using two long $X M M$ Newton observations in 2013 November and 2014 June and found no correlation during the two modes. This result suggests a different origin for the bulk of the emission in the UV/optical bands, likely from the asymmetrically heated face of the companion star (Papitto et al., in prep.; note that the optical pulsed emission discovered by Ambrosino et al. 2017 plays only a minor role in the optical budget, as it shows a fractional amplitude always below 1 percent). Unfortunately, the X-ray flares observed during our campaign were not covered by Swift UVOT, precluding a comparison between the X-ray and UV emissions during the flaring mode. We note that (Bogdanov et al. 2015) found a significant correlation for the flaring mode, with some flaring episodes preceding those in the X-rays and vice versa, and having time delays of a few hundred seconds.

The new observations provide an updated characterisation of the high- and low-mode X-ray spectra, and confirm and constrain the main properties of our model (see Campana et al. 2016). In particular, we found a smaller variation of the disc black body component normalization across the high and low modes with respect to our previous data modelling (see Table 4). This parameter can be related to the magnetospheric radius, at which the pressure of the NS magnetosphere equals the ram pressure of the inflowing matter. We found that in the high mode, the magnetospheric radius attains a value $r_{m}=26_{-9}^{+11}$ $\mathrm{km}$, whereas in the low mode only a lower limit $r_{m}>185$ $\mathrm{km}$ can be inferred. It is typically assumed that the inner edge of the accretion disc is truncated at the magnetospheric radius. Therefore, in the high mode, the inner disc radius is fully consistent within the uncertainties with lying between the corotation radius $r_{c o r}=\left(G M P^{2} / 4 \pi^{2}\right)^{1 / 3} \sim 24 \mathrm{~km}$, at which matter in Keplerian orbit corotates with the NS, and the light cylinder radius $r_{l c}=c P / 2 \pi \sim 81 \mathrm{~km}$, at which a particle corotating with the magnetosphere would move at the speed of light (here $P$ is the NS spin period and $c$ the speed of light). In the low mode, the inner disc radius is instead pushed beyond the light cylinder radius. In the framework of the basic theory for the accretion regimes in NS LMXBs (see e.g. Campana et al. 1998), these results further corroborate our scenario where the repeated transitions between the two modes are due to fast switches between a weak propeller regime in the high mode ( $\left.r_{c o r} \lesssim r_{m}<r_{l c}\right)$ and the radio pulsar mechanism in the low mode $\left(r_{m}>r_{l c}\right)$. The observed radio brightening associated with the X-ray low mode (Bogdanov et al. 2017) can be easily reconciled with our model, because an enhancement of the radio emission is naturally expected when the inflowing matter is rapidly ejected by the radiation pressure of the pulsar.

The transitions between the different accretion regimes onto MSPs were recently explored by Parfrey \& Tchekhovskoy (2017), who performed the first time-dependent generalrelativistic magnetohydrodynamics simulations of the accretion of magnetized plasma onto a rotating, magnetized NS. Interestingly, their simulations produce a peculiar regime where the accretion flow spends most of the time inside the light cylinder (i.e. the propeller regime), but is regularly expelled out through it (i.e. the radio pulsar ejection regime via the pulsar wind). The simulations show that these transitions can take place over a few seconds (K. Parfrey, priv. comm.). This theoretical result is again fully compatible with our scenario for the mode switching of J1023, with the fact that the system is observed most of the time in the high mode (propeller) and only a minority of the time in the low mode (particle ejection in the form of pulsar wind), and with the short timescale observed for the mode switching.

The fractional amplitude of the optical pulsations recently discovered from the system showed variations in time up to a factor of $\sim 4$ on timescales of $\sim 1.2 \mathrm{ks}$, and upper limits were reported during a few time spans (Ambrosino et al. 2017; see in particular their Fig. 2). The lack of simultaneous X-ray observations prevented the authors from clarifying if the appearance/disappearance of the optical pulses is strictly related to the mode switching. According to our scenario, optical pulsations should be observed only in the low mode if these are due to synchrotron emission by relativistic electrons and positrons in the magnetosphere of a rotation-powered pulsar, as proposed by Ambrosino et al. (2017). On the other hand, detection of optical pulsed emission only in the high mode (i.e. simultaneously with the X-ray pulsations) would require that a different emission mechanism is at work, possibly related to residual channelled accretion onto the NS polar caps in the weak propeller regime. The maximum pulsed luminosity achievable in the optical band via cyclotron emission by thermal electrons located within the accretion columns, $L_{c y c l} \sim 3 \times 10^{29} \mathrm{erg} \mathrm{s}^{-1}$, is smaller than the maximum observed pulsed luminosity, $L_{\text {opt,puls }} \sim 10^{31} \mathrm{erg} \mathrm{s}^{-1}$, only by a factor of $\sim 30$ (Ambrosino et al. 2017). A beamed cyclotron emission pattern with beaming factor $b=L_{c y c l} / L_{\text {opt,puls }} \sim 1 / 30$ cannot be ruled out a priori, and might be in principle a viable possibility (assuming that the maximum optical pulsed luminosity is attained during the high mode; otherwise $b$ might be even larger). If instead optical pulsations are detected both in the low and high modes and they arise indeed from the two above-mentioned different processes, we shall expect to observe some differences in the pulse profile shape and amplitude across the two modes in the optical. Alternatively, an intriguing possibility is that a radio pulsar dipole spin-down mechanism might be constantly at work (as already suggested by Coti Zelati et al. 2014; Stappers et al. 2014; Takata et al. 2014), regardless of the source state, converting a 
tiny fraction $\left(\sim 2 \times 10^{-5}\right)$ of the spin-down power into optical pulsed emission. In all the above-mentioned scenarios, the simplest explanation for the non-detection of the rotation-powered radio pulsations would be that these are overwhelmed by the accreting matter during the propeller regime in the high mode, and enshrouded by a large amount of intervening ionized material around the NS during the particle ejection regime in the low mode. Simultaneous X-ray and optical observations with high time resolution and large enough photon counting statistics are necessary to single out the different modes of J1023 in the optical band, characterize accurately the optical pulsations as a function of the luminosity modes, and test our predictions.

We also performed a detailed high-resolution X-ray spectroscopy of the system using all RGS data acquired on J1023 since it became much brighter in the X-rays in 2013. This allowed us to identify several narrow features in the soft X-ray spectrum. A commonly used diagnostic for the emitting regions can be derived from the ratio between the different components of the Helium-like triplets (Porquet \& Dubau 2000). In particular, a first-order estimate of the density of the emitting gas can be inferred from the parameter $R=z /(x+y)$, which quantifies the ratio between the intensity of the forbidden line $(z)$ and that of the sum of the intercombination emission $(x+y)$. For the case of $\mathrm{J} 1023$, the best constraint on $R$ is provided by the $\mathrm{N}$ VI triplet, which shows a prominent intercombination emission but lacks the forbidden line. Assuming that the emission lines originate in a pure photo-ionized plasma, this implies a high-density plasma, with electron density $n_{e} \gtrsim 10^{11} \mathrm{~cm}^{-3}$ (see e.g. Fig. 8 of Porquet \& Dubau 2000). This lower limit is consistent with the emission lines being produced within the accretion disc.

A detailed study of possible correlations and lags between the UV, optical, and near infrared emissions on timescales of seconds was impossible, owing to a non-optimal setup of the $X M M-N e w t o n$ OM and bad weather conditions that impeded ground-based observations. However, this analysis will be the object of future work thanks to approved and ongoing multiwavelength campaigns.

Acknowledgements. We thank Kyle Parfrey and Amruta Jaodand for helpful discussions, and Alessandro Papitto and Domitilla de Martino for comments on the manuscript. We thank the referee for useful comments and suggestions. We acknowledge the International Space Science Institute (ISSI) that funded and hosted the international team "The disk-magnetosphere interaction around transitional millisecond pulsars", and we thank all the members of the team for fruitful discussions. FCZ acknowledges Università dell'Insubria and the Anton Pannekoek Institute for Astronomy of the University of Amsterdam for their kind hospitality during the time that some of this work was carried out. The scientific results reported in this study are based on observations obtained with XMM-Newton, NuSTAR and Swift. XMM-Newton is an ESA science mission with instruments and contributions directly funded by ESA Member States and the National Aeronautics and Space Administration (NASA). The NUSTAR mission is a project led by the California Insitute of Technology, managed by the Jet Propulsion Laboratory, and funded by NASA The Neil Gehrels Swift Observatory is a NASA/UK/ASI mission. The XMM Newton SAS is developed and maintained by the Science Operations Centre at the European Space Astronomy Centre. The NuSTAR Data Analysis Software (NuSTARDAS) is jointly developed by the ASI Science Data Center (ASDC, Italy) and the California Institute of Technology (Caltech, USA). This research has made use of softwares and tools provided by the High Energy Astrophysics Science Archive Research Center (HEASARC) Online Service, which is a service of the Astrophysics Science Division at NASA/GSFC and the High Energy Astrophysics Division of SAO. FCZ, NR and DFT are supported by grants AYA2015-71042-P and SGR2014-1073. SC acknowledges support from the ASI/NuSTAR NARO16 funds. NR acknowledges funding in the framework of the Netherlands Organization for Scientific Research (NWO) Vidi award number 639.042.321.

\section{References}

Aliu, E., Archambault, S., Archer, A. et al. 2016, ApJ, 831, 193

Alpar, M. A., Cheng, A. F., Ruderman, M. A., \& Shaham, J. 1982, Nature, 300, 728

Ambrosino, F., Papitto, A., Stella, L., et al. 2017, Nat. Astron., 1, 266

Archibald, A. M., Stairs, I. H., Ransom, S. M., et al. 2009, Science, 324, 1411

Archibald, A. M., Kaspi, V. M., Hessels, J. W. T., et al. 2013 , ArXiv e-prints [arXiv: 1311.5161]

Archibald, A. M., Bogdanov, S., Patruno, A., et al. 2015, ApJ, 807, 62

Arnaud, K. A. 1996, in Astronomical Data Analysis Software and Systems V, eds. G. H. Jacoby, \& J. Barnes (San Francisco: ASP), ASP Conf. Ser., 101, 17 Baglio, M. C., D’Avanzo, P., Campana, S., et al. 2016, A\&A, 591, A101

Bassa, C. G., Patruno, A., Hessels, J. W. T., et al. 2014, MNRAS, 441, 1825

Bhattacharya, D., \& van den Heuvel E. P. J. 1991, Phys. Rep., 203, 1

Bogdanov, S., Archibald, A. M., Bassa, C., et al. 2015, ApJ, 806, 148

Bogdanov, S., Deller, A., Miller-Jones, J. C. A., et al. 2017, ApJ, submitted, [arXiv: 1709.08574]

Breeveld, A. A., Landsman W., Holland S. T., et al. 2011, Gamma Ray Bursts 2010, eds. J. E. McEnery, J. L. Racusin, \& N. Gehrels (New York: AIP), AIP Conf. Proc., 1358, 373

Burrows, D. N., Hill, J. E., Nousek J. A.. et al. 2005, Space Sci. Rev., 120, 165

Campana, S., Colpi, M., Mereghetti, S., Stella, L., \& Tavani, M. 1998, A\&ARv, 8,279

Campana, S., Coti Zelati, F., Papitto, A., et al. 2016, A\&A, 594, A31

Coti Zelati, F., Baglio, M. C., Campana, S., et al. 2014, MNRAS, 444, 1783

Deller, A. T., Archibald, A. M., Brisken, W. F., et al. 2012, ApJ, 756, L25

Deller, A. T., Moldon, J., Miller-Jones, J. C. A., et al. 2015, ApJ, 809, 13

de Martino, D., Falanga, M., Bonnet-Bidaud, J.-M., et al. 2010, A\&A, 515, A25

de Martino, D., Belloni, T., Falanga, M., et al. 2013, A\&A, 550, A89

den Herder, J. W., Brinkman, A. C., Kahn, S. M., et al. 2001, A\&A, 365, L7

Engström, L., \& Litzén, U. 1995, J. Phys. B At. Mol. Phys., 28, 2565

Gu, M. F., Schmidt, M., Beiersdorfer, P., et al. 2005, ApJ, 627, 1066

Hakala, P., \& Kajava, J. J. E. 2018, MNRAS, 474, 3297

Halpern, J. P., Gaidos, E., Sheffield, A., Price-Whelan, A. M., \& Bogdanov, S. 2013, Astron. Telegram, 5514, 1

Harrison, F. A., Craig, W. W., Christensen, F. E., et al. 2013, ApJ, 770, 103

Heinke, C. O., Rybicki, G. B., Narayan, R., \& Grindlay, J. E. 2006, ApJ, 644, 1090

Jaodand, A., Archibald, A. M., Hessels, J. W. T. et al. 2016, ApJ, 830, 122

Kallman, T., Liedahl, D., Osterheld, A., Goldstein, W., \& Kahn, S. 1996, ApJ, 465,994

Li, K. L., Kong, A. K. H., Takata, J. et al. 2014, ApJ, 797, 111

Linares, M. 2014, ApJ, 795, 72

Mason, K. O., Breeveld, A., Much, R., et al. 2001, A\&A, 365, L36

McConnell, O., Callanan, P. J., Kennedy, M., et al. 2015, MNRAS, 451 3468

Papitto, A., \& Torres, D. F. 2015, ApJ, 807, 33

Papitto, A., Ferrigno, C., Bozzo, E., et al. 2013, Nature, 501, 517

Parfrey, K., \& Tchekhovskoy, A. 2017, ApJ, 851, L34

Patruno, A., Archibald, A. M., Hessels, J. W. T., et al. 2014, ApJ, 781, L3

Poole, T. S., Breeveld, A. A., Page, M. J., et al. 2008, MNRAS, 383, 627

Porquet, D., \& Dubau, J. 2000, A\&AS, 143, 495

Pradhan, A. K., Chen, G. X., Delahaye, F., et al. 2003, MNRAS, 341, 1268

Radhakrishnan, V., \& Srinivasan, G. 1982, Curr. Sci., 51, 1096

Roming, P. W. A., Kennedy, T. E., Mason, K. O., et al. 2005, SSRv, 120, 95

Shahbaz, T., Linares, M., Nevado, S. P., et al. 2015, MNRAS, 453, 3461

Smith, R. K., \& Brickhouse, N. S. 2014, Adv. At. Mol. Opt. Phys., 63, 271

Srinivasan, G. 2010, New A Rev., 54, 93

Stappers, B. W., Archibald, A. M., Hessels, J. W. T., et al. 2014, ApJ, 790, 39

Strüder, L., Briel, U., Dennerl, K., et al. 2001, A\&A, 365, 18

Taam, R. E., \& van den Heuvel, E. P. J. 1986, ApJ, 305, 235

Takata, J., Li, K. L., Leung, G. C. K., et al. 2014, ApJ, 785, 131

Tauris, T. M., \& van den Heuvel, E. P. J. 2006, in Compact Stellar X-Ray Sources, eds. W. H. G. Lewin, M. van der Klis (Cambridge: Cambridge Univ. Press), 623

Tavani, M., \& Arons, J. 1997, ApJ, 477, 439

Tendulkar, S. P., Yang, C., An, H., et al. 2014, ApJ, 791, 77

Torres, D. F., Ji, L., Li, J., et al. 2017, ApJ, 836, 68

Turner, M. J. L., Abbey, A., Arnaud, M., et al. 2001, A\&A, 365, L27

Wik, D. R., Hornstrup, A., Molendi, S. et al. 2014, ApJ, 792, 48

Wilms, J., Allen, A., \& McCray, R. 2000, ApJ, 542, 914 